\title{
Sociocultural considerations in aging men's health: implications and recommendations for the clinician
}

\section{Keywords}

Sociocultural factors

Men

Aging

Patient-physician relations

Illness behaviors

Cultural competency
Nathan S. Consedine, PhD

Department of Psychology, Long Island University, USA

Anne Skamai, PhD Department of Family Medicine, SUNY Downstate Medical Center, USA

\section{E-mail:}

anne.skamai@downstate. edu

Online 15 August 2009

\section{Nathan S. Consedine and Anne Skamai}

Abstract

The health of aging men, and the particular health concerns that they confront, are commanding greater attention within clinical medicine. Remnants of the biomedical tradition that examines prevention, diagnosis, and treatment of disease states in isolation from the historical, developmental, and cultural contexts in which they occur still predominate even though their impact on the medical care context is slowly becoming recognized. The relations of sociocultural characteristics to patterns of disease risk, health behaviors, symptom responses, delayed diagnosis, and treatment adherence are less welldocumented or understood. Empirical evidence supports the role of sociocultural factors in the understanding and management of health among aging men. The essential features of specific ethno-cultural, psychosocial, socio-demographic, and health system variables can offer practical clinical applications for providers. Awareness and understanding of such characteristics will facilitate culturally competent practices that are more likely to engage men in collaborative educational, disease management, and health maintenance efforts throughout their lifespan. (c) 2009 WPMH GmbH. Published by Elsevier Ireland Ltd.

An evolving body of evidence regarding the particular health issues confronting populations of aging men is receiving increased recognition from practitioners [1]. Ill-health in later life results from complex multi-factorial processes that originate from a combination of biological, social, and psychological factors, all of which are experienced, interpreted, and responded to within men's sociocultural frameworks. While many practitioners acknowledge the importance of such factors within a biopsychosocial medical model, the reality of overwhelming clinical demands can prompt a more biomedical approach in practice. This results in a tendency to examine and treat disease with minimized attention to related, but often more ambiguous, factors. Although recent work suggests that gender differences in self-reported health may be smaller than previously thought [2], a significant literature implies that while women become disabled with age, men experience acute events [3]. Men die, on average, between 6 and 7 years earlier than women, and have higher death rates at all ages and for most leading causes of death. Such disparities are receiving increasing attention $[1,4]$.

Less well-recognized in clinical practice is the fact that the health issues confronting older men as well as the characteristics that place them at risk are unequally distributed across ethnic groups. National United States (U.S.) data describing incidence and mortality for leading killers suggest that African American men are more likely to die of heart disease, most cancers, cerebrovascular disease, and diabetes [5]. Similarly, in comparison to European Americans 
(172.9/100 000) and Hispanics (127.6/100 000), African American men (275.3/100 000) have the highest incidence of prostate cancer in the United States [6]. African Americans have more than twice the mortality rate of Whites for prostate cancer [7], and among Hispanics it remains the second leading cause of cancer death [8]. Minority men are more likely to present with metastatic cancer/distant stage cancers [5], reducing both treatment options and survival rates. Compounding this problem is an ongoing tendency to examine individuals from highly heterogeneous backgrounds as if they were similar. So, for example, while many "African American" or "Black" men have been in the United States for generations, more recent immigrant groups from the Caribbean and Africa were raised, and live in, cultural environments that are near-completely distinct [9].

\section{Characterizing diverse groups of aging men}

Broadly speaking, there are three ways in which researchers have conceptualized disparities across groups. The first - that groupings represent biologically distinct sets of individuals with differing disease vulnerabilities has generally fallen into disfavor. Second, public health and epidemiological traditions suggest that ethnicity serves as a proxy for sociodemographic and environmental disadvantages including lower income, education/ social class, insurance coverage, employment characteristics, access to foodstuffs and health resources, environmental exposures and so on. Although important, there are reasons to think that this characterization may oversimplify the nature of ethnic "belongingness", not least because research shows that ethnic disparities in behavior and outcome persist even when such variables are controlled.

The third approach is more ethnographic and psychological and encompasses characteristics such as culture, identity, minority status, immigration history, religion, language, kinship, and place of origin [10] as well as more psychological constructs such as normative patterns of relating, emotion and emotion regulation, health beliefs and illness cognitions [11]. In this view, understanding the links between ethnicity and outcome requires that we tease apart the meaning of ethnicity as a variable by identifying the proximal variables through which it impacts outcomes [9]. This view suggests that the way disease, illness, and healthrelated symptoms develop, occur, and are experienced are shaped by a man's contexts. So, while disease has its origins in biological and developmentally-acquired patterns of exposure and risk, such patterns are socioculturally embedded. For instance, early dietary patterns and neuro-endocrine reactivity that may exacerbate a hereditary predisposition for midlife diabetes onset [12] could be influenced by social and environmental considerations including financial or geographic restrictions in access to healthy foods, caregiver availability for balanced meal preparation, and stress responses to neighborhood violence, domestic traumas, immigration troubles, or unemployment concerns. Cultural or familial beliefs about the urgency of treating "sugar" could further delay initial or subsequent presentation for treatment. Symptoms and diseases as well as their management are experienced and interpreted within particular contexts suggesting that engagement with an aging man on the matter of his health requires an understanding and appreciation of where he is coming from.

An important implication of this perspective is that both the health and general functioning of current cohorts of aging men represent the "culmination" of a particular set of experiences that have implications for their health. Some experiences are particular to men that are currently old (rather than those who will become old), while some reflect ideas about masculinity or aging more generally. It has been suggested, for example, that persons raised in the United States during the Great Depression spent a powerfully formative period living with economic hardship [3]. Such experiences have likely made them hardier, more stoic and with a belief in the value of work, and more closely linked to their families. Older men tend to have low tolerance for uncertainty or medical intimacy, value independence and control, individualism, and "toughing it out," are often isolated, have poor self care, and are frequently avoidant in managing stressors. Men from under-represented groups are, if anything, "more masculine" in these regards; they generally report (even) lower anxiety [13], have reduced health-related self-efficacy [14], and, although gender-specific 
data are lacking, are likely more fatalistic about disease and mortality [15].

\section{Implications for clinical work with diverse groups of aging men}

Consideration of variation in sociocultural characteristics should inform personalized clinical prevention, diagnosis, and intervention initiatives for men in medical settings. Unfortunately, the bulk of the available literature is rooted in anthropological, sociological, epidemiological, and psychological frameworks; fewer clinical medicine studies directly identify sociocultural factors or report related interventions for men. Furthermore, clinical recommendations for aging men regarding modifications of healthcare delivery are sparse.

Sociocultural characteristics impact expectations, understanding, and communication between patients and providers. Relevant considerations range from psychosocial characteristics among men that contribute to disease development, to male-particular patterns of health-promoting and health-deleterious behaviors, as well as symptom sensitivity and responding tendencies, to health screening practices, treatment decision-making, and adherence behavior. The challenge of culturally-competent care is to bridge these intersections through attention to men's unique situations and through increased clinician awareness of their own reflexive culture- or gender-specific assumptions and biases within the patient-provider relationship.

\section{Risk factors for disease incidence}

There are three broad classes of phenomena that can impact disease risk in diverse groups of aging men. First, there is evidence that distinct groups vary in genetic and biological disease predispositions. Among aging men, for example, prostate cancer incidence is higher among Blacks, and even higher among immigrants from the Caribbean [16]. Similar differences in incidence and mortality have been observed among subpopulations of Asian Americans [17]. Second, disparities in health behaviors linked to disease are relatively welldocumented. In contrast to Whites, minority men (particularly those of African American or Asian/Pacific Islander heritage) are more likely to be obese, have dietary practices involving larger and less nutritious meals, exercise less frequently when older, and are more likely to smoke [18]. Similarly, although smoking rates are declining in older groups, tobacco has been used in most cultures [19] and has historically been viewed as acceptable for males, together with drinking alcohol in many (although not all) societies. Cultural variations in the specific methods and patterns of tobacco use may still bear consequences for their current health status, even among men who no longer smoke. The causes of these ethnic and gender disparities are complex and appear to further interact with aspects of social class. For example, while body mass index (BMI) has a negative linear relation with income or education among women, the link between income or education and BMI varies by birthplace among men [20,21]. Care should be taken in considering such factors among immigrant groups who tend to increase BMI as a function of duration of residence [22].

Third, and generally less well appreciated by clinicians, are the psychosocial characteristics that (1) vary across gender, cultural, and ethnic lines and (2) may create risks for bodily responses linked to disease risk. In contrast to women, for example, men report greater hostility and anger, both of which are robust predictors of cardiovascular issues, with effect sizes similar to those of smoking and hypertension [23]. Although self-reported negative emotionality, stress, and depression are generally lower among minority men, low reporting may represent either emotional restriction or avoidance [10]. Patterns of avoidant coping are dangerous to health with one study among HIV patients finding that avoidance was associated with nearly twice the rate of decline in CD4+ counts and viral load [24]. Alternately, stress may tend to manifest in "self-medicating" behaviors. African American men, for instance, may attempt to "self-medicate" with drink, cigarettes, and "comfort foods" to protect their mental, at the expense of their physical, health [25].

Culturally-sensitive clinical assessment should address the modifiable and non-modifiable risk factors that may predispose male patients from different backgrounds to increased disease and illness vulnerabilities (see Table 1). Clinicians who identify and discuss such vulnerabilities in a straightforward manner, with interested attention toward the sociocultural origins and contexts in which 
Table 1 Sociocultural risk considerations in men's vulnerability to illness

\begin{tabular}{ll} 
& Modifiable \\
\hline 1. Biological and genetic reactivity - e.g., aggressive tumor biology & $\sqrt{ }$ \\
2. Lifestyle behaviors - e.g., diet, physical activity, smoking & $\sqrt{ }$ \\
3. Exposure to stressful life experiences - e.g., immigration, job loss & $\sqrt{ }$ \\
4. Environmental exposure to toxic agents - e.g., worksite chemicals & $\sqrt{ }$ \\
5. Psychological predisposition - e.g., health beliefs, emotional regulation style, self-efficacy & \\
\hline Adapted from [16] and [30]. &
\end{tabular}

men operate may be more successful at engaging their patients in prevention and health management plans $[26,27]$.

\section{What is "health"? Discrepancies between men and their providers?}

Men and their providers often differ in their conceptions of what defines a state of "health." While physicians may focus on the presence or prevention of chronic disease and associated physical or cognitive impairments, [28] men's subjective sense of their own health and wellbeing is typically less "medical" or easily defined. While both groups value the absence of illness, the preservation of masculine role functioning (e.g., working) may have a greater bearing on perceptions of self-rated health even in the presence of physical symptoms or chronic disease $[29,30]$.
Collaborative, health-promoting relationships between men and their providers can unify differing views through setting meaningful agendas for time-limited office visits [4]. Given that men perceive, interpret, and respond to illness through the socio- and ethno-cultural lenses of their experience, clinicians can identify and negotiate differences in goals of care by determining what is important to the patient [31]. In one Australian study, for example, men above 50 expressed concerns about "breaking down" as they aged but also expressed concern regarding the quality of their intimate relationships during visits to their General Practitioner [32]. Mutual goalsetting may feel unfamiliar or uncomfortable within certain cultures and for individuals who have pressing current circumstances [33]. Similarly, the provider's focus on a chronic problem with invisible symptoms can easily be eclipsed by a man's more immedi-

Table 2 Questions to assess a sociocultural explanatory model for health conditions

Preventive or well visits

How do people in your culture view men's health? Do you see your health like this?

How does the health of your body and mind matter in your life?

What do men in your culture do to prevent illness from coming on?

Do you consider yourself well or ill? How would you describe your health?

Diagnostic or treatment visits

What do you believe caused your symptoms/condition?

What do you believe about the cause of your symptoms/condition? How do people in your culture understand this kind of problem?

How serious is the problem? What course do you expect it to take?

What do you think this problem does inside your body?

What do you fear most about this condition? What would be the worst outcome?

What have you or others done to manage this situation?

What do you dislike or fear most about the treatment for this condition?

Psychosocial considerations

How do family members or important others view this condition and/or treatment?

How does your condition affect your everyday life (self-care, work, leisure)?

How does your health or condition affect family? Friends? Others who care for you?

What result do you hope for from this healthcare visit/relationship?

Adapted from $[35,37,87]$. 
ate social and family concerns [34]. Consequently, care plans should attend to men's "explanatory models" for their health issues (see Table 2) [35]. A provider need not sacrifice either medical care goals or much time in order to engage men in effective agenda setting. However, providers need to be alert for impulses to dismiss or minimize a man's concerns that may initially appear trivial and be willing to probe for a better understanding of his unique sociocultural values or challenges. For example, specific follow-up questions to a man's vague mention of "many people" in the home could reveal stressful living conditions that impact his sleep, eating, finances and, therefore, his ability to engage in health maintenance. A sensitive and non-judgmental attitude from the provider is key to help avoid feelings of shame in the patient at not being able to solve the problem or admitting that he has one in the first place [36].

\section{Symptom sensitivity and reporting}

Symptom recognition, which depends to some extent on cultural definitions of health, may be a primary step in the "health-seeking" process for aging men [37]. Significant proportions of health issues are detected because of patient concerns regarding symptoms [38], yet this common impetus for a physician visit is, to an extent, dependent on the patient's inclination or ability to detect bodily changes. A lack of symptom visibility, or feeling "well," has been implicated as a significant predictor of the likelihood to neglect preventive care or discontinue self-management efforts among Hispanic and African-American men $[39,40]$. Data from the Canadian Heart Health Survey, for example, suggest that $43 \%$ of men (and 33\% of women) previously diagnosed with high blood pressure stated lack of awareness about their hypertensive status. [41] Similarly, although men have been undersampled in cross-ethnic studies of cancer pain [42] men are generally less sensitive to bodily pain [43], a factor that may contribute to their reduced likelihood of presenting with pain conditions (e.g., migraines or fibromyalgia) [44].

Older men are typically low in both anxiety and depressed affect, two conditions that elevate symptom reporting and increase the motivation or the ability to detect bodily changes [45]. It may be that older men are less attuned to bodily changes and/or less willing to admit to discomfort and thus unlikely to seek assistance. However, even given symptom detection, a host of considerations often prevent people from seeking assistance [46]. Specifically, a lack of care-seeking may be further moderated by cultural stigma and other frameworks through which symptoms are interpreted [47]. Among aging men in the United Kingdom, delays in care have been attributed to challenges of limited vocabulary to address potentially embarrassing symptoms, fear of having a health concern judged as trivial, or desire to avoid a negative response if a problem lacks clear or simple resolution [36]. Limited patience to make or wait for appointments and a reluctance to sit in a waiting room with women and children may further fuel avoidance [36]. Furthermore, help-seeking in response to symptoms requires that the individual interpret them as sufficiently troublesome or label them as serious enough to warrant action [48]. Men are less likely than women to seek help for problems including depression, substance abuse, physical disabil-

Table 3 Reframing male "help-seeking" as "health-seeking" behaviors

1. Emphasize specific health behaviors as empowering; highlight benefits

2. Tie present behaviors to future outcomes; relate to man's current life situation and values

3. Choose active versus passive terms - e.g., using health resources vs. getting help

4. Use masculine, 'action-based' metaphors - e.g., check and change the oil regularly -before the light goes on or engine seizes

5. Provide physiological or medicalized versus mental or stigmatized explanations of stress to encourage attention to anxiety, depression, and other health-compromising emotions

6. Change attention patterns to body sensations and disease-related changes by 'prescribing' and reinforcing regular self-monitoring of selected physiological reactions and symptoms

7. Facilitate trust and healthcare utilization by sensitivity to potential access factors - e.g., offer 'after work' clinic hours when possible

Adapted from $[36,47,64]$. 
ities and stressful life events [49]. Given generational tendencies toward stoicism, avoidance, and individualism - what we might term "traditional masculine behavior" [49] these problems could be exacerbated among older men. Table 3 offers some potential suggestions to assist men to reframe physical symptoms and reconstruct help-seeking as desirable, health-seeking behavior.

\section{Screening behaviors}

Characteristics particular to the aging male may contribute to the low rates of screening for various diseases, particularly colorectal and prostate cancers. Screening rates are sub-recommendation among most men ( $\mathrm{ca}$. $50 \%$ of recommendation), but are particularly low among minority men, recent immigrants, those with low education and/or those without health insurance [50]. The absence of symptoms, as discussed in the preceding sections, can lend clinicians insight into the origins of infrequent preventive behaviors. As noted, clinicians should be cautious when interpreting studies that rely on census categories to distinguish groups because meaningful differences between subgroups may be missed. For example, while prostate screening beliefs and behaviors among African American men have received considerable attention, more recent work suggests the presence of key subpopulation differences. Among 344 U.S.-born and immigrant men from seven distinct ethnic groups, differences in coping predicted differences in prostate-specific antigen (PSA) testing rates that were obscured with analyses utilizing White, Black, and Hispanic categories [51].

Clinicians should also consider how sociocultural characteristics may be particularly relevant to particular examinations. For example, although actual discomfort is minimal, digital rectal examinations (DRE) require that man bend forward and allow a doctor (often another male who may be younger) to insert a lubricated finger into his anus. Unsurprisingly, DRE screens are associated with anxiety and embarrassment as well as concerns regarding vulnerability, sexuality, and humiliation [16]. The salience of such feelings as a screening barrier may differ across groups. One study of colorectal screening among Vietnamese, Latinos and Whites found that perceived discomfort and embarrassment predicted lower screening only among Latinos [52]. Given that older men have highly "traditional" gender roles [3], and are likely aversive to situations in which they lack control or that are sexually ambiguous, sensitivity to these considerations may encourage men in this group to face such aversion. Additional psychosocial screening barriers that likely vary across cultural contexts and may trigger men's tendency for stress- or threat-induced avoidance include fear of cancer [16], under- or over-estimated perception of risk, and mistrust of the medical system [40]. Finally, inadequate knowledge [53], poor understanding of risks and benefits [52], and little or no access to insurance, especially among recent immigrants, require continued attention in screening for chronic conditions such as hypertension, diabetes, and cancer.

Evidence continues to affirm the importance of proactive physician education and recommendation of screening examinations for men across diverse groups. When provided with direct physician communication about the need for screening, $61.2 \%$ of black American men reported DRE screening even though they "didn't like it" [54]. Although clinical trial findings are equivocal regarding the efficacy of prostate screening, minority men are underrepresented in these studies [55] and media reports can further complicate men's under-

Table 4 Screening communication considerations among socioculturally diverse men

1. Deliver a clear recommendation message based on risk and encourage questions

2. Tailor communication to individual risk, health beliefs, and environmental context

3. Provide culturally-relevant oral and written information at appropriate health literacy levels

4. Be alert to screening hesitation related to fear or mistrust of healthcare system

5. Elicit support for men's screening from religious or community leaders - e.g., consider having a letter of endorsement from respected leaders on hand

6. Be knowledgeable about institutional screening policies and assist with alternate access to screening as needed

Adapted from [54]. 
standing of risk profiles and screening needs. Such complexity underscores the key role of the physician in communicating recommendations based on individualized risk profiles. Specific considerations to tailor effective communication are summarized in Table 4 [54].

\section{Treatment decision-making}

Given developments in treatment science, older men confronted with diagnoses such as heart disease and, particularly, cancer, must distill a bewildering array of information, advice, demands, and treatment options. In addition to grappling with economic and logistic considerations, older men must "weigh up" complex and often-conflicting data circumscribing clinical efficacy and longevity and the likelihood of unpleasant (and unmanly) short- and long-term side effects (e.g., hair loss, weight gain). Complex decision-making tasks are where the influence of sociocultural factors becomes most strongly evident [56]. Evidence describes considerable variability in older adults' willingness to discuss life-support decisions given a metastatic cancer diagnosis in a multiethnic U.S. population of European, African, Korean, and Mexican Americans [57], and the particular medical situation will often influence the desired degree of participation in medical decisions [30].

Patterns of emotionality and stress, as well as health beliefs, are implicated in patterns of treatment decision-making [45]. Although data regarding men are scanty, increased anxiety tends to predict a greater likelihood of opting for medical interventions [58]; recent evidence suggests prostate-cancer anxiety is a strong predictor of how long men wait to initiate androgen deprivation therapy (ADT) following biochemical recurrence, with more anxious men more likely to more rapidly initiate ADT [59].

Given such evidence, both the degree and the source of men's disease/treatment anxieties can be telling, as several health behaviors are influenced by health-related anxieties [16]. Generally, low anxiety and high avoidance in older men coupled with the high disease fatalism seen among minorities [15] may place aging men at high risk for inaction in instances where treatment-related decisions are urgently needed. Men are constitutionally accustomed to (and prefer) situations in which they are independent and "in control" and may become passive in circumstances that are threatening or beyond their control. Clinicians should seek to identify such characteristics early when treating aging minority men, accept the possibility that some men may appear disinterested while others will simply desire to be "told what to do" $[60,61]$, and be sure to supplement primary pharmacological treatments with psycho-educational programming specifically designed to counteract or capitalize upon characteristics of the aging male. One study found that African American and Latino men with diabetes were more satisfied with greater physician facilitation of decision-making, even when they had initially stated a lower desire for participation [62]. Among a large population of veterans, however, effective physician communication and sufficient patient understanding of information was found to be more significant in the promotion of diabetic self-management than the degree of active patient participation in decision-making [63]. In discussions about necessary treatment decisions, it may be advantageous to capitalize on men's desires for independence, anxiety-reduction, and control by highlighting and clearly explaining how they can be proactive in their own treatment, especially with regard to behavioral and self-management strategies. Evidence suggests that if men are given some power to negotiate with the clinician on specific aspects of lifestyle change (e.g., reductions in high-sodium foods for hypertension [20] or appointment scheduling for diabetes [64]), this can result in good rapport, and even partial adherence accrues some benefit.

The clinician's ability to elicit and provide information in a manner that the patient prefers and understands may be more a critical skill with men of varied ethnicities than their participation style in making care decisions [40]. Health information that is tailored in wording, image, and tone to fit with cultural beliefs, identity, practices, language, and literacy skills is essential to effective patient education (see Table 5) $[33,65]$. Although multiracial, Black, and Hispanic adults are disproportionately represented - as are men - among those with low health literacy, attention to health literacy levels has been found to reduce racial/ethnicity disparities in self-rated health status and some preventive health behaviors 
Table 5 Tailor information to cultural and linguistic needs through relevant channels

Examples of Culturally and Linguistically Appropriate Services (CLAS) include:

Oral communications

Speak slowly

Choose familiar words; avoid medical jargon

Use "teach-back" and "show me" methods: "What do you understand about...?" or "Show me how you would give yourself the insulin."

Provide staff support - e.g., for filling out forms

Provide trained interpreters for men with low English proficiency; if unsure of need, ask

Print educational materials

Use visual aids to reinforce education during office visits - e.g., draw pictures, use anatomical models

Edit pamphlets to 5 th grade reading level; check readability with word-processing tools

Simplify amount of text and number of ideas; increase images and "white space" in materials

Provide specific recommendations with descriptive pictures that address misperceptions, exact

measurements

- e.g., illustrate "one beer" $=8$ oz can, not 40 oz bottle

Check translations of materials for contextual and dialect discrepancies

Culturally-concordant channels

Network members

Appeal to health beliefs and target health information both for men and key members of social network

- e.g., elicit spousal beliefs about and support for food preparation and medical visits

Vignettes

Use patient stories to illustrate points about particular health risks and health syndromes specific to diverse

"older men"

Models (media, physician, trained peer supports)

Consider in-clinic video education with culturally-concordant male modeling of healthy masculine attributes

who access assistance for problems; follow up with discussion

Refer to respected or popular public figures as models for men's prevention and adherence behaviors

Adapted from $[33,68,85]$.

among older adults [66,67]. Clinicians should actively consider low health literacy as a factor in a man's lack of participation during clinical encounters and take extra steps to minimize shame or avoidance of medical encounters by encouraging questions, checking patient understanding, and including family members in the education process [68].

\section{Adherence to medication and lifestyle change regimens}

Adherence to chronic pharmacological therapies is poor [69], a problem that is recognized internationally [70]. Studies indicate that adherence to statin regimens, for example, is around $40 \%$ with elderly patients being less likely to adhere [71]. Issues particular to groups of aging males reveal similar problems. One study of 2,640 men on Medicaid in California, for example, found that only $40 \%$ were adherent with any benign prostatic hyperplasia
(BPH) medication [72]. There are indications that adherence to pharmaceutical regimens is lower among men than it is among women [73]. Adherence also appears to be generally lower among Black men, including those taking antihypertensives [73], HIV medication [74], and inhaled asthma steroids [75].

Although physician recommendation practices, biological treatment-response variations, toxicities, and economic and logistical concerns, are important, poor adherence is fundamentally a behavioral problem [76]. While the literature linking psychosocial characteristics to adherence among aging males is small, adherence studies in other areas provide some guidance. Characteristics particular to the aging male such as restricted emotion regulatory characteristics or avoidant coping predict lower adherence [77], as do levels of practical and emotional social support, family cohesiveness or conflict, marital status, and living arrangements [78]. Reduced contact with network members (a characteristic of 
Table 6 Potential social network interventions to engage men "where they live"

1. Include spouse or family members in clinic visits to engage, educate, and empower as agents in man's health management

2. Create "resource maps" of men's social networks (family, friends, religious, community) with delineated roles and contact information; distribute to network members

3. Invite healthy male patient or spouse of chronically ill male patient to co-deliver talk about lifestyle change or health management at senior centers, community centers or churches

4. Partner with administrators in senior or community centers, church-based health ministries, etc to implement on-site lifestyle changes - e.g., reduced sodium in community meals, walking program, chronic illness support groups

5. Partner with a nutritionist and local grocers to run a clinic-based "healthy food choices" event and encourage local availability of healthy food products

Adapted from [64,80,83,84].

aging men) predicts reduced compliance with lipid-lowering medications [79] and it has been suggested that family members may prefer to cope with the illness threat by concentrating on practical issues [80].

The origins of non-adherence may differ between men and women and, indeed, may vary among men from different ethnic groups. Work among African American men examining the origins of appointment keeping and medication adherence suggest that a host of characteristics including the meaning of health, blood pressure, and medication, their social contexts, and the patient-provider relationship are important in making lasting change [81]. Assessment of complimentary and alternative medicine use among men from diverse cultures may reveal adjunctive treatment behaviors, such as traditional dietary, herbal, or body- or faith-based healing approaches, could contribute to drug interaction effects or indicate unrecognized emotional distress [82].

Patient-centered exploration of barriers to a man's treatment adherence on a case by case basis offers the best possibility for negotiating behavior change which, if at all, will often progress in a small, step-wise fashion. It is important to bear in mind that many factors within a patient's socio-cultural context may not be amenable to change. Likewise, the potential benefits or threats to health may not be given the same weight as the perceived 'costs' of the proposed change, e.g., allocation of a family's strained finances for medications or extra effort of a spouse for special food preparation. Paradoxically, it may be a provider's willingness to understand, respect, and accept a patient's current level of readiness or confidence to change that can strengthen trust and rapport and open the way to change in the future.

In contrast to women, tendencies towards avoidance, repression, and social isolation in older men may contribute to greater nonadherence. Older men who are widowed or divorced - men tend to relate to networks via their wives [83] - along with those currently managing other forms of stress (e.g., financial) may be at particular risk. Where appropriate, it may behove clinicians to involve spouses or adult children in medication and behavioral adherence interventions with aging men [80]. Where this is not possible (or desirable), physicians may suggest that men partner with other friends or relatives or implement a system of telephonic or mail-based reminders. Finally, it should be noted that attention to such characteristics may be particularly important when treating minority men whose social networks differ both structurally as well as functionally (Table 6); domestic arrangements in such networks - that may also vary as a function of immigration status are more likely to involve multiple generations and a greater value is placed on both kin relationships and the provision of assistance $[9,84]$.

\section{"Know thyself:" clinician-specific considerations}

The concept of 'cultural competence' has become a popular term in clinical medicine, but how does one actually do it? In addition to patient-centered strategies, informed both by men's individual life situations and available evidence, the fundamental premise and challenge of cultural competence includes 
Table 7 Clinician guidelines for self-assessment of cultural assumptions

1. Consider how personal cultural assumptions about men's health behavior affects approach to male patients

2. Explore men's life and health in sociocultural context with respectful curiosity

3. Check de facto expectations of patient behavior based on sociocultural stereotypes or biases

4. Make an extra effort to elicit clarifying information with open-ended questions

5. Modify style of interaction and expectations of patient participation based on cultural preferences Adapted from $[85,86,88]$.

Table 8 Evidence-based sociocultural recommendations for older men

\begin{tabular}{|c|c|}
\hline & References \\
\hline $\begin{array}{l}\text { 1. Careful assessment of modifiable and non-modifiable sociocultural risk factors } \\
\text { can help clinicians target the most salient prevention and intervention plan for men }\end{array}$ & 16,30 \\
\hline $\begin{array}{l}\text { 2. Identify men's relevant health beliefs and values to tailor culturally-relevant } \\
\text { health management plans }\end{array}$ & $61,64,87$ \\
\hline $\begin{array}{l}\text { 3. Reframe health-supportive behaviors as strengths within culturally-consistent } \\
\text { constructs of masculinity and self-efficacy }\end{array}$ & $4,30,36,47$ \\
\hline $\begin{array}{l}\text { 4. Provide clear, direct, and patient-centered physician communication } \\
\text { about screening needs }\end{array}$ & 40,54 \\
\hline $\begin{array}{l}\text { 5. Tailor health literate prevention, treatment, and adherence messages } \\
\text { through socioculturally consistent channels }\end{array}$ & $20,33,63,64,68,83$ \\
\hline $\begin{array}{l}\text { 6. Create health-responsive opportunities for men through engaging } \\
\text { social networks in clinic, work, and community-based sites }\end{array}$ & $4,64,65,80,83,84$ \\
\hline $\begin{array}{l}\text { 7. Be self-reflective and flexible as a clinician to gender- and culture-based } \\
\text { assumptions about health behaviors among diverse groups }\end{array}$ & $36,60,61,85,86,88$ \\
\hline
\end{tabular}

clinicians' reflective self-assessment of sociocultural beliefs so as not to perpetuate stereotypes that become yet another barrier to care $[85,86]$. The clinician's interpersonal awareness, combined with a flexible attitude of open-minded inquiry, creative problem-solving, willingness to accommodate differences and respect for patient choices is culturallycompetent care (Table 7).

\section{Conclusion}

Although cultural competence objectives have become standard in medical training, skill implementation in practice is often inadequate and may appear daunting given the pressures of healthcare delivery environments [87]. While integrated healthcare system change is needed at organization and community levels, a growing base of evidence consistently highlights the clinician's role in facilitating improved communication, satisfaction, adherence, and health outcomes through culturally-tailored approaches $[33,84,88]$. In this regard, the clinician is a critical agent of change toward health promotion and quality of life for older men.

Table 8 summarizes available evidence-based clinical recommendations to guide practice. Working collaboratively with patients, clinicians can create opportunities to focus prevention and intervention efforts on those factors that will optimize "successful" aging within each man's unique sociocultural context.

\section{References}

[1] Eckert JK, Rubinstein RL. Older men's health - sociocultural and ecological perspectives. Med Clin N Am 1999;83(5):1151.

[2] Arber S, Cooper H. Gender differences in health in later life: the new paradox? Soc Sci Med 1999;48(1):61-76.

[3] Consedine NS, Magai C, Krivoshekova YS. Sex and age cohort differences in patterns of socioemotional functioning in older adults and their links to physical resilience. Ageing Int 2005;30(3):209-43.

[4] Sadovsky R. Men's healthcare needs improvement: a recommendation for a midlife men's health assessment visit. jmhg 2005;2(3):375-81.

[5] ACS. Cancer facts and figures for African Americans, 2007-2008. Atlanta, GA: American Cancer Society, 2007.
[6] Jemal A, Murray T, Samuels A, Ghafoor A, Ward E, Thun MJ. Cancer statistics, 2003. CA Cancer J Clin 2003;53(1):5-26.

[7] ACS. Cancer facts and figures, 2004. New York: American Cancer Society, 2004.

[8] O'Brien K, Cokkinides V, Jemal A, Cardinez CJ, Murray T, Samuels A, et al. Cancer statistics for Hispanics, 2003. CA Cancer J Clin 2003;53(4):208-26. 
[9] Consedine NS, Magai C, Conway F. Predicting ethnic variation in adaptation to later life: styles of socioemotional functioning and constrained heterotypy. J Cross-Cult Gerontol 2004;19:95-129.

[10] Consedine NS, Magai C, Kudadjie-Gyamfi EK, Kaluk Longfellow J, Ungar TM, et al. Stress versus discrete negative emotion in the prediction of physical complaints: does predictive utility vary across groups. Cult Divers Ethnic Minority Psychol 2006;12(3):541-57.

[11] Walter FM, Emery J, Braithwaite D, Marteau TM. Lay understanding of familial risk for common chronic diseases: a systematic review and synthesis of qualitative research. Ann Fam Med 2004;2(6):583-94.

[12] Peng H, Hagopian W. Environmental factors in the development of Type 1 diabetes. Rev Endocr Metabol Disord 2006;7(3):149-62.

[13] Consedine NS, Magai C. The uncharted waters of emotion: ethnicity, trait emotion and emotion expression in older adults. J Cross-Cult Gerontol 2002;17:71-100.

[14] Siegel K, Karus D, Schrimshaw EW. Racial differences in attitudes toward protease inhibitors among older HIV-infected men. AIDS Care - Psychol Socio-Med Aspects AIDS/HIV 2000;12(4):423-34.

[15] Powe BD. Fatalism among elderly African Americans: effects on colorectal cancer screening. Cancer Nurs 1995;18(5):385-92.

[16] Consedine NS, Adjei BA, Ramirez PM, McKiernan J. An object lesson: differences in source determine the relations that trait anxiety, prostate cancer worry, and fear of screening hold with prostate screening frequency. Cancer Epidemiol Biomarkers Prevent 2008;17(7):1631-9.

[17] McCracken $M$, Olsen $M$, Chen Jr MS, Jemal A, Thun M, Cokkinides V, et al. Cancer incidence, mortality, and associated risk factors among Asian Americans of Chinese, Filipino, Vietnamese, Korean, and Japanese ethnicities. CA Cancer J Clin 2007;57(4.

[18] Myers HF, Kagawa-Singer M, Kumanyika SK, Lex BW, Markides KS. Panel III: Behavioral risk factors related to chronic diseases in ethnic minorities. Health Psychol 1995;14:613-21.

[19] Anwar S, Williams SA. Cultural aspects of tobacco use. Dental Update 2008;35(1):3840: 43-44, 47.

[20] Han H, Kim K, Kang J, Jeong S, Kim EY, Kim MT. Knowledge, beliefs, and behaviors about hypertension control among middle-aged Korean Americans with hypertension. J Commun Health 2007;32(5):324-42.

[21] Sanchez-Vaznaugh EV, Kawachi I, Subramanian SV, Sanchez BN, Acevedo-Garcia D. Do socioeconomic gradients in body mass index vary by race/ethnicity, gender, and birthplace? Am J Epidemiol 2009;169(9): 1102-12.
[22] Goel MS, McCarthy EP, Phillips RS, Wee CC. Obesity among US immigrant subgroups by duration of residence. J Am Med Assoc 2004;292(23):2860-7.

[23] Consedine NS, Magai C, Chin S. Hostility and anxiety fear differentially predict coronary heart disease in men and women. Sex Roles 2004;50(1-2):63-75.

[24] Ironson G, O'Cleirigh C, Fletcher MA, Laurenceau JP, Balbin E, Klimas N, et al. Psychosocial factors predict CD4 and viral load change in men and women with human immunodeficiency virus in the era of highly active antiretroviral treatment. Psychosom Med 2005;67(6):1013-21.

[25] Jackson JS. What can resolve the paradox of mental health disparities. APA Monitor Psychol 2002;33:46.

[26] Teal CR, Street RL. Critical elements of culturally competent communication in the medical encounter: a review and model. Soc Sci Med 2009;68(3):533-43.

[27] Stewart AL, Nápoles-Springer A, PérezStable EJ, Posner SF, Bindman AB, Pinderhughes $\mathrm{HL}$, et al. Interpersonal processes of care in diverse populations. Milbank Quart 1999;77(3):305-39.

[28] Wilcox BJ, He Q, Chen R. Midlife risk factors and healthy survival in men. J Am Med Assoc 2006;296(16):2343-50.

[29] Naslindh-Yispangar A, Silivonen M, Kekki P Health, utilization of health service, 'core' information, and reasons for non-participation: a triangulation study amongst nonrespondents. J Clin Nurs 2008;17:2972-8.

[30] Verbruge L. Gender and health: an update on hypotheses and evidence. J Health Soc Behav 1985;26(3):156-82.

[31] Brown VA, Bartholomew K, Naik AD. Management of chronic hypertension in older men: an exploration of patient goal-setting Patient Educat Counseling 2007;69:93-9.

[32] Hall RH. Promoting men's health. Aust Fam Physician 2003;32(6):401-7.

[33] Kreuter MW, Lukwago SN, Buckholtz DC, Clark E, Sanders-Thompson V. Achieving cultural appropriateness in health promotion programs: targeted and tailored approaches. Health Educ Behav 2003;30(2):133-46.

[34] Brown RL, Baumann LJ, Helberg CP, Han $Y$, Fontana SA, Love RR. The simultaneous analysis of patient, physician and group practice influences on annual mammography performance. Soc Sci Med 1996; 43:315-24.

[35] Kleinman A. The illness narratives: Suffering, healing, and the human condition. New York: Basic Books; 1988.

[36] White A, Banks I. Help seeking in men and the problems of late life diagnosis. 2nd edition In: Kirby R, Carson C, Kirby M, Farah R, editors. Men's health, London: Martin Dunitz and Parthenon Publishing; 2004: p. 1-7.
[37] Chrisman N. The health seeking process: an approach to the natural history of illness. Cult Med Psychiatr 1977;62:291-8.

[38] Consedine NS, Krivoshekova YS, Harris CR. Bodily embarrassment and judgment concern as separable factors in the measurement of medical embarrassment: psychometric development and links to treatment-seeking outcomes. Br J Health Psychol 2007;12: 439-62.

[39] Brown C, Schulberg HC, Madonia MJ. Clinical presentations of major depression by African Americans and Whites in primary medical care practice. J Affect Disord 1996;41:181-91.

[40] Shavers VL, Underwood W, Moser RP. Race/ ethnicity and the perception of the risk of developing cancer. Am J Prevent Med 2009;37(1):64-7.

[41] Kirkland SA, MacLean DR, Langille DB, Joffres MR, MacPherson KM, Andreou P. Knowledge and awareness of risk factors for cardiovascular disease among Canadians 55 to 74 years of age: results from the Canadian Heart Health Surveys, 19861992. Can Med Assoc J 1999;161(8 Supp. 3):S10-6.

[42] Im E, Chee W, Guevara E, Liu Y, Lim H, Tsai $H$, et al. Gender and ethnic differences in cancer pain experience: a multiethnic survey in the United States. Nurs Res 2007; 56(5):296-306

[43] Klatzkin RR, Mechlin B, Girdler SS. Menstrual cycle phase does not influence gender differences in experimental pain sensitivity Eur J Pain 2009: in press.

[44] Fillingim RB, Maixner W. Gender differences in the responses to noxious stimuli. Pain Forum 1995;4(4):209-21.

[45] Consedine NS, Moscowitz JT. The role of discrete emotions in health outcomes: a critical review. J Appl Prevent Psychol 2007;12:59-75.

[46] Shaw C, Williams K, Assassa PR, Jackson C. Patient satisfaction with urodynamics: a qualitative study. J Adv Nurs 2000;32(6): 1356-63.

[47] Moller-Leimkuhier AM. Barriers to helpseeking by men: a review of the sociocultural and clinical literature with particular reference to depression. J Affect Disord 2002;71:1-9.

[48] Leventhal $H$, Diefenbach $M$, Leventhal EA. Illness cognition: using common sense to understand treatment adherence and affect cognition interactions. Cog Therapy Res 1992;16(2):143-63.

[49] Galdas PM, Cheater F, Marshall P. Men and health help-seeking behaviour: literature review. J Adv Nurs 2005;49(6): 616-23.

[50] ACS, Cancer prevention and early detection, 2007-2008. New York: American Cancer Society; 2007. 
[51] Kudadjie-Gyamfi EK, Consedine NS, Magai C. On the importance of being ethnic: coping with the threat of prostate cancer in relation to prostate cancer screening. Cult Divers Ethnic Minority Psychol 2006;12(3): 509-26.

[52] Walsh JME, Kaplan CP, Nguyen B, Gildengorin G, McPhee SJ, Perez-Stable EJ. Barriers to colorectal cancer screening in Latino and Vietnamese Americans. J Gen Intern Med 2004;19:156-66.

[53] Consedine NS, Christie MA, Neugut Al. Physician, affective, and cognitive variables differentially predict "initiation" versus "maintenance" PSA screening profiles in diverse groups of men. Br J Health Psychol 2009;14:303-22.

[54] Woods VD, Montgomery SB, Herring RP, Gardner RW, Stokols D. Social ecological predictors of prostate-specific antigen blood test and digital rectal examination in Black American men. J Natl Med Assoc 2006;98(4):492-504.

[55] Andriole GL, Crawford ED, Grubb RL, Buys SS, Chia D, Church TR, et al. Mortality results from a randomised prostate-cancer screening trial. New Engl J Med 2009;360(17): 1310-9.

[56] Slovic P, Peters E, Finucane ML, MacGregor DG. Affect, risk, and decision making. Health Psychol 2005;24(4):S35-40.

[57] Blackhall LJ, Frank G, Murphy ST, Michel V, Palmer JM, Azen SP. Ethnicity and attitudes towards life sustaining technology. Soc Sci Med 1999;48(12):1779-89.

[58] Bober SL, Hoke LA, Duda RB, Regan MM, Tung NM. Decision-making about tamoxifen in women at high risk for breast cancer: clinical and psychological factors. J Clin Oncol 2004;22(24):4951-7.

[59] Dale W, Hemmerich J, Bylow K, Mohile S, Mullaney M, Stadler WM. Patient anxiety about prostate cancer independently predicts early initiation of androgen deprivation therapy for biochemical recurrence in older men: a prospective cohort study. J Clin Oncol 2009;27(10):1557-63.

[60] Gordon HS, Street RI, Scharf BF, Souchek J. Racial differences in doctors' informationgiving and patients' participation. Cancer 2006;107(6):1313-20.

[61] de Haes H. Dilemmas in patient centeredness and shared decision making: a case for vulnerability. Patient Educ Counseling 2006;62:291-8.

[62] Golin C, DiMatteo R, Duan N, Leaky B, Gelbert I. Impoverished diabetic patients whose doctors facilitate their participation in medical decision making are more satisfied with their care. J Gen Intern Med 2002; 17:866-75.

[63] Heisler M, Bouknight RR, Hayward RA, Smith DM, Kerr EA. The relative importance of physician communication, participatory decision making, and patient understanding in diabetic self management. J Gen Intern Med 2002;17(4):243-52.

[64] Lerman I, Lozano L, Villa AR, HernándezJiménez $S$, Weinger $K$, Caballero $A E$, et al. Psychosocial factors associated with poor diabetes self-care management in a specialized Center in Mexico City. Biomed Pharmacother 2004;58(10):566-70.

[65] Brach C, Fraserirector I. Can cultural competency reduce racial and ethnic health disparities? Med Care Res Rev 2000;57: 181-217.

[66] Bennett IM, Chen J, Soroui JS, White S. The contribution of health literacy to disparities in self-related health status and preventive health behaviors in older adults. Ann Family Med 2009;7(3):204-11.

[67] Kutner M, Greenberg E, Jin Y, Paulsen C. The health literacy of America's adults: results from the 2003 National Assessment of Adult Literacy. New York: National Center for Education Statistics; 2006.

[68] Davis TC, Williams MV, Branch WT, Green $\mathrm{KW}$. Explaining illness to patients with limited literacy. In: Whaley BB, editor. Explaining illness. Mahwah, NJ: Lawrence Erlbaum Associates; 2000

[69] Lee JK, Grace KA, Taylor AJ. Effect of a pharmacy care program on medication adherence and persistence, blood pressure, and low-density lipoprotein cholesterol: a randomized controlled trial. J Am Med Assoc 2006;296(21):2563-71.

[70] Wens J, Vermeire E, Van Royen P, Sabbe B, Denekens J. GPs' perspectives of type 2 diabetes patients' adherence to treatment: a qualitative analysis of barriers and solutions. BMC Family Practice 2005;6.

[71] Jackevicius CA, Mamdani M, Tu JV. Adherence with statin therapy in elderly patients with and without acute coronary syndromes. J Am Med Assoc 2002;288(4) 462-7.

[72] Nichol MB, Knight TK, Wu J, Barron R, Penson DF. Evaluating use patterns of and adherence to medications for benign prostatic hyperplasia. J Urol 2009;181(5): 2214-22.

[73] Hyre AD, Krousel-Wood MA, Muntner $P$, Kawasaki L, DeSalvo KB. Prevalence and predictors of poor antihypertensive medication adherence in an urban health clinic setting. J Clin Hypertension 2007;9(3):179-86.

[74] Kalichman SC, Ramachandran B, Catz S. Adherence to combination antiretroviral therapies in HIV patients of low health literacy. J Gen Intern Med 1999;14(5): 267-73.

[75] Apter AJ, Boston RC, George M, Norfleet AL, Tenhave T, Coyne JC, et al. Modifiable barriers to adherence to inhaled steroids among adults with asthma: it's not just black and white. J Allergy Clin Immunol 2003; 111(6):1219-26.

[76] Magai C, Consedine NS, Neugut Al, Herschman DL. Commmon psychosocial factors underlying breast cancer screening and breast cancer treatment adherence: a conceptual review and synthesis. J Women's Health 2007;16(1):11-23.

[77] Pereira DB, Antoni MH, Danielson A, Simon T, Efantis-Potter J, O'Sullivan MJ. Inhibited interpersonal coping style predicts poorer adherence to scheduled clinic visits in human immunodeficiency virus infected women at risk for cervical cancer. Ann Behav Med 2004;28(3):195-202.

[78] DiMatteo MR. Social support and patient adherence to medical treatment: a metaanalysis. Health Psychol 2004;23(2):207-18.

[79] Kaplan RC, Bhalodkar NC, Brown EJ, Wflite J, Brown DL. Race, ethnicity, and sociocultural characteristics predict noncompliance with lipid-lowering medications. Prevent Med 2004;39(6):1249-55.

[80] Gallant MP, Spitze GD, Prohaska TR. Help or hindrance? How family and friends influence chronic illness self-management in older adults Res Aging 2007;29:375-409.

[81] Rose LE, Kim TK, Dennison CR, Hill MN. The contexts of adherence for African Americans with high blood pressure. J Advan Nurs 2000;32(2):587-94.

[82] Lee MM, Chang JS, Jacobs B, Wrensch MR. Complimentary and alternative medicine use among men with prostate cancer in four ethnic populations. Am J Public Health 2002;92(10):1606-9.

[83] Consedine NS, Fiori KL. Gender moderates the associations between attachment and discrete emotions in middle age and later life. Aging Mental Health 2009: in press.

[84] Anderson LM, Scrimshaw SC, Fullilove MT, Fielding JE. Task Force on Community Preventive Services. The Community Guide's model for linking the social environment to health. Am J Prevent Med 2003;24(9S): 12-20.

[85] United States Department of Health and Human Services - Office of Minority Health. A physician's practical guide to culturally competent care. Office of Minority Health, 2007.

[86] American Medical Association Council on Ethical and Judicial Affairs. Gender disparities in medical decision making, 1992.

[87] Kleinman A, Benson P. Anthropology in the clinic: the problem of cultural competency and how to fix it. PLoS Med 2006.

[88] Betancourt JR, Green AR, Carillo EJ. Defining cultural competence: a practical framework for addressing racial/ethnic disparities in heatlh and health care. Public Health Rep 2003:3:293-302 